\title{
Directed differentiation protocols for successful human intestinal organoids derived from multiple induced pluripotent stem cell lines
}

\author{
Amanda L. Kauffman ${ }^{1}$, Jason E. Ekert ${ }^{1}$, Alexandra V. Gyurdieva ${ }^{1}$, Michael A. Rycyzyn ${ }^{1}$ and Pamela J. Hornby ${ }^{2 *}$ \\ *Correspondence: phornby@its.jnj.com

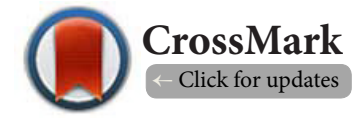 \\ 'Biologics Research, Biotechnology CoE, Janssen Pharmaceutical J\&J, Spring House, PA 19477, USA. \\ ${ }^{2}$ Cardiovascular Metabolic Research, Janssen Pharmaceutical J\&J, Spring House, PA 19477, USA.
}

\begin{abstract}
Background: Relatively little has been reported comparing the ability of different induced pluripotent stem cells (iPSCs) and protocols to derive human intestinal organoids (HIO), although there is potential to supply HIO for translational research and regenerative medicine. In view of the time and effort required to differentiate HIO, protocols for differentiation were compared and five iPSC lines, produced by retroviral or non-viral reprogramming methods, were concurrently differentiated to HIO to evaluate the robustness and repeatability of using cellular markers by flow cytometry and immunohistochemistry.

Methods: iPSCs were differentiated to definitive endoderm by Activin A treatment (Protocol I) or Myostatin (GDF8), GSK-3 $\beta$ inhibitor (CHIR99021) and B27 supplement (Protocol II) and then differentiated to hindgut using FGF4 and WNT3A (Protocol I), or KGF and Retinoic Acid (Protocol II) resulting in spheroids that were differentiated to HIO in 3-dimensional culture in matrigel containing EGF, Noggin, and R-Spondin1.

Results: Definitive endoderm markers (CXCR4 and SOX17) were similar using both protocols and in all cell lines, but in their absence, spheroids to not develop. HIO derived from excised hindgut spheroids (Protocol II) showed more consistent expression of epithelial markers E-Cadherin, CDX2, villin, and chromogranin A. Morphological characteristics such as budding are predictive of robust enterocyte differentiation (villin positive) in HIO.

Conclusions: Substituting different members of the growth factor families (e.g., TGF $\beta$ ) is equally or more effective for producing epithelial lineages in HIO. Although there are no early quantitative predictors of level of success, all reprogrammed somatic cells could be differentiated to produce HIO. Several morphological characteristics (excisable spheroids and budding in HIO) were associated with HIO success. This could improve cost-effectiveness and ease of differentiation as HIO become more commonly available as translational $3 \mathrm{D}$ models of the gut.
\end{abstract}

Keywords: Induced pluripotent stem cell, intestine, spheroid, human intestinal organoid

\section{Introduction}

The advent of induced pluripotent stem cells (iPSCs) has opened up new avenues for generating human cell and tissue-specific organoids in vitro, by reprogramming adult somatic cells to an embryonic stem cell-like state for use in directed differentiation [1]. Directed differentiation of human intestinal organoids (HIO) from iPSC has been accomplished by several labs [2-7] including ourselves [8]. These protocols require a series of growth factors defined to promote the differentiation of Definitive Endoderm (DE) that form hindgut spheroids and then human $\mathrm{HIO}$ in 3D culture. There are some differences in the iPSC differentiation protocols to $\mathrm{HIO}$ from these labs. Examples are in initial differentiation to DE by treatment with TGF $\beta$ family Activin A with low increasing serum [9], where Activin A treatment can 
Kauffman et al. Stem Cell Biology and Research 2015,

http://www.hoajonline.com/journals/pdf/2054-717X-2-1.pdf

doi: 10.7243/2054-717X-2-1

be replaced by the TGF $\beta$ family molecule Myostatin (GDF8) in the presence of GSK-3 $\beta$ inhibitor CHIR99021 (GSK3i) and B27 Supplement (B27), to produce more mature DE cells [10]. An additional difference is the DE transition to hindgut cells by treatment with posterior patterning growth factors FGF-4 [11] and WNT [12] in one protocol [5] or Keratinocyte Growth Factor/FGF-7 (KGF) and Retinoic Acid (RA) $[13,14]$ to mimic epithelial-mesenchymal interactions in a modified protocol [8]. KGF was previously shown to promote intestinal differentiation and proliferation in human intestinal epithelial cell models $[15,16]$. Retinoic acid (RA) is an upstream regulator of the intestine-specific transcription factor Caudal type homeobox 2 (CDX2) [17], the hindgut epithelial transcription factor [18]. Hindgut spheroids expressing CDX2, are further developed into $\mathrm{HIO}$ within $3 \mathrm{D}$ matrigel containing intestinal growth and morphogenesis factors Noggin, R-Spondin 1, and EGF $[4,8]$. A difference, not obvious to someone not performing these protocols, is that spheroids budding off from the adherent sheet of differentiating cells can be either floating $[4,5]$ or adherent $[8]$ that are selected for 3D culture.

$\mathrm{HIO}$ have the potential to change the study of human intestinal epithelia, by surmounting the limited accessibility of human ex vivo samples and avoiding use of $2 \mathrm{D}$ cell lines for permeability and transport assays used to understand therapeutic delivery and efficacy. In addition, HIO provide the opportunity to derive multicellular systems with normal or disease phenotype in order to understand the emerging science around the host gut metabolism. Since reproducibility and standardization of protocols are critical for iPSC emerging science, we initially compared these protocols by immunohistochemical localization and expression of several epithelial markers in $\mathrm{HIO}$. While doing this, we noticed differences in handling of the differentiating tissue and environment could result in different outcomes. We had noted generally higher levels of epithelial cell lineage marker gene expression markers in 3D HIO compared to those differentiated in 2D transwell culture [8]. In the present study, we found that excised attached spheroids, which were reminiscent of crypt structures, had more pronounced CDX2 immunostaining compared to collected floating spheroids. These two observations suggested to us that changes in handling and localization of structures, could drive the outcome to successful differenti- ated $\mathrm{HIO}$, maybe as much as the differentiation growth factor protocols. Finally, iPSC lines can be produced by the forced expression of reprogramming transcription factors (e.g., Oct4, Sox2, KIf4, and c-Myc) by means of retroviral transduction [1] although this can potentially disrupt cellular stability and function [19]. Thus, to compare the robustness of intestinal differentiation, five cell lines were contemporaneously differentiated and their morphology and a number of markers of differentiation through $\mathrm{HIO}$ tracked. Since some cell lines failed to appropriately differentiate with markers associated with each stage, or differed in the level of expression, another aim was to determine if there were any predictive markers that would reduce time spent unsuccessfully deriving $\mathrm{HIO}$. A direct comparison of the HIO differentiation of iPSC reprogrammed from different somatic cell types and by different reprogramming methodology has not been reported, to our knowledge. In view of the investment to make $\mathrm{HIO}$, including the recent successful in vivo engraftment and maturation of $\mathrm{HIO}$ in mouse, [6], it is important to address the likelihood of finding biomarkers of successful differentiation as early as possible. A portion of this work has been published in abstract form [20].

\section{Methods}

\section{Cells used}

iPSCs: The iPSC lines used in this comparative study were derived from various cell types of origin and reprogramming methods (Table 1). The iPSC lines were all generated by transducing genetically unmodified human cells with viruses individually encoding the human transcription factors (OCT4, SOX2, KLF4, C-MYC) and, for non-retroviral modified RNA or mini-circle DNA, c-MYC was omitted and additional transcription factors were used, as indicated (Table 1). Original mesenchymal stem cells were isolated and obtained from System Bioscience or within J\&J and reported elsewhere [21]. In general, iPSCs were transitioned from Mouse Embryonic Fibroblast co-culture to feeder-free culture conditioned upon freezing. Differentiation protocol modifications were compared initially on retroviral reprogrammed human kidney-derived cells (A1145A) and foreskin fibroblast-derived cells (D2043A and F1089A). Subsequently karyotyping revealed abnormalities in F1089A and it was replaced by another retroviral re-

Table 1. iPSC lines derived cell types and retroviral or non-viral reprogramming methods.

\begin{tabular}{llll}
\hline iPSC line & Source & Original cell type & Reprogramming method \\
\hline A1145A & J\&J & Kidney-Derived Cells & Retroviral: OCT4, SOX2, KLF4, c-MYC \\
B2198A & J\&J & Kidney-Derived Cells & Modified RNA: OCT4, KLF4, SOX2, C-MYC, LIN2 \\
C2128A & J\&J & Umbilical Tissue-Derived Cells & Modified RNA: OCT4, KLF4, SOX2, C-MYC, LIN2 \\
D2043A & System & Normal Foreskin Fibroblasts & Retroviral: OCT4, SOX2, KLF4, c-MYC \\
& Biosciences & & \\
E2055A & System & Adipocyte Stem Cells & Mini-Circle DNA: OCT4, SOX2, LIN28, NANOG \\
& Biosciences & & \\
F1089A & J\&J & Normal Foreskin Fibroblasts & Retroviral: OCT4, SOX2, KLF4, c-MYC \\
\hline
\end{tabular}


programmed cell (D2043A). The optimized protocol was then compared during contemporaneous differentiation of cell lines, which included three additional cell lines reprogrammed by "integration free" techniques utilizing modified RNA or minicircle DNA [22,23]-kidney-derived (B2198A) umbilical tissue-derived cells (C2128A) and pre-adipocyte-derived cells (E2055A).

iPSC lines were maintained in mTeSR 1 culture media (STEMCELL Technologies; Vancouver, BC) on culture dishes/ flasks coated with embryonic stem cell (ES)-qualified Geltrex (Life Technologies; Carlsbad, CA) and passaged by Dispase (STEMCELL Technologies) dissociation every 3-5 days as previously described [4].

\section{iPSC differentiation}

Induced pluripotent stem cells were seeded into ES-qualified Geltrex-coated 12- or 24-well tissue culture plates as cell clumps at a density of $5-7.5 \times 10^{4} \mathrm{cell} / \mathrm{s} / \mathrm{cm}^{2}$. Differentiation was started 3 days later, when cells typically reached $60-90 \%$ "confluency" without any morphological indications of spontaneous differentiation. A summary of the protocols are shown (Table 2) is Protocol I [4,5] and modified Protocol II [8]. The key modifications from Protocol 1 were as follows: In place of Activin A, iPSCs were differentiated for 3 days using $500 \mathrm{ng} / \mathrm{mL}$ GDF8 (R\&D Systems; Minneapolis, MN) and $1.5 \mu \mathrm{M}$ Stemolecule ${ }^{\mathrm{TM}}$ CHIR99021 (GSK-3 inhibitor, StemGent; Cambridge, MA) as previously reported, with $0.1 \%$ Fatty-acid Free Bovine Serum Albumin (FAF-BSA, Proliant/Lampire; Pipersville, PA) and the addition of 1XB27 Supplement (Life Technologies) in RPMI 1640 (Life Technologies). In place of 3-4 days in WNT3A and FGF-4, Stage 1 cells were further differentiated for 4 days in Advanced DMEM/F12 (Life Technologies) with $50 \mathrm{ng} / \mathrm{mL}$ KGF (Peprotech; Rocky Hill, NJ), 0.1\% FAF-BSA, 15 mM HEPES (Life Technologies), and 1XB27 Supplement, followed by an additional 3-day hindgut differentiation step to Stage 2 with the addition of $2 \mathrm{mM}$ Retinoic Acid (RA; Sigma-Aldrich; St. Louis, MO), 1:200 Insulin, Transferrin, Selenium, Ethanolamine Solution (Life Technologies), and 2\% FAF-BSA.

Finally, another difference from Protocol $I$ is that in addition to floating spheroid structures (collected in Protocol I) there were those that formed within the adherent layer of cells at Stage 2 in Protocol II. Therefore to test the ability of forming $\mathrm{HIO}$, initially both floating and excised adherent spheroids were collected using a P200 pipette tip to $15 \mathrm{~mL}$ conical tubes with media and spheroids allowed to settle by gravity. Subsequently for Stage 3 differentiation, spheroids were treated the same for both protocol I and II in 3D intestinal matrigel culture and differentiated to Stage 3 for 26-36 days. Media was changed daily until Stage 3 differentiation, when media was changed every 3-4 days. Stage 3 organoids were passaged every 2-3 weeks, as previously described [4].

\section{Protein markers of differentiation}

Cell surface markers and transcription factor expression were quantified by flow cytometry. Undifferentiated or Stage 1 clusters and cells were detached by treatment with Accutase (Sigma-Aldrich; St. Louis, MO) and stained for viability using Near Infrared Live/Dead kit (Invitrogen; Carlsbad, CA) before fixation in Cytofix Buffer (BD Biosciences; San Jose, CA). Cells were then stained with $\mathrm{PE}$-conjugated mouse anti-human CD184/CXCR4 (Biolegend; San Diego, CA) before permeabilized with Phosflow Perm Buffer (BD Biosciences) and stained with APC-conjugated goat polyclonal anti-human SOX17 (R\&D Systems; Minneapolis, MN). Stained cells were analyzed in conjunction with appropriate compensation controls (BD Biosciences) by flow cytometry using an LSR Fortessa FACS Sorter (BD Biosciences). Raw data was analyzed by FlowJo analysis software (Tree Star; Ashland, OR).

Tissue morphology and protein localization were determined in $\mathrm{HIOs}$ by immunohistochemistry. Floating spheroids and $\mathrm{HIOs}$ were transferred from matrigel, settled by gravity, fixed in 10\% Neutral Buffered Formalin (ThermoScientific; Rockford, IL) and embedded in paraffin. Sections $(4 \mu \mathrm{m})$ of $\mathrm{HIO}$ or human tissue bank (MTB330, QualTek Molecular Labs; Goleta, CA) were dried on glass slides $\left(60^{\circ} \mathrm{C}\right)$. Steam heat induced epitope recovery (SHIER) system [24] was followed by were incubated in mouse anti-human E-Cadherin (M3612, Dako; Denmark), CDX2 (M3636, Dako), villin (M3637, Dako), chromogranin A or control (non-immune) IgG and Avidin Biotin Complex immunohistochemistry (BioTek Solutions/Ventana Medical Systems).

Table 2. Summary of protocol I and modified protocol II for differentiation of HIO.

\begin{tabular}{llllll}
\hline & iPSC & Stage 1 & Stage 2 & $\begin{array}{l}\text { Stage 3 (from stage 2 floating or excised } \\
\text { spheroids) }\end{array}$ \\
\hline $\begin{array}{l}\text { Protocol I } \\
\text { growth factors }\end{array}$ & -- & Activin A & $\begin{array}{l}\text { WNT3A, -- } \\
\text { FGF-4 }\end{array}$ & EGF, Noggin, R-Spondin1 \\
\hline $\begin{array}{l}\text { Protocol II } \\
\text { growth factors }\end{array}$ & -- & GDF-8, GSKi, & KGF & KGF, RA & EGF, Noggin, R-Spondin1 \\
\hline Days incubation & -- & B27 & & & \\
\hline Cell type & Pluripotent & Definitive & & Hindgut & Intestinal epithelial \\
\hline Stage markers & OCT4, SOX2, Nanog, & SOX17, & & CDX2 & E-Cadherin, CDX2, Villin, chromagranin A \\
& SSEA3,Tra-1-81 & CXCR4 & & & \\
\hline
\end{tabular}


Kauffman et al. Stem Cell Biology and Research 2015,

http://www.hoajonline.com/journals/pdf/2054-717X-2-1.pdf

Semi quantitative analysis of the immunohistochemical staining intensity and frequency was performed. Samples were scored separately for each of the three antibodies tested and each received a membrane score for E-cadherin, a nuclear score for CDX2, and both a cytoplasmic and apical score for villin. Each score is arrived at by a semi-quantification technique in which the reviewer estimates the percent of cells that are at a $0,1+, 2+$, or $3+$ staining intensity (where $0=$ no staining, $1+$ $=$ low intensity staining, $2+=$ moderate intensity staining and $3+=$ high intensity staining). A sample was considered positive if staining of $10 \%$ of cells at $1+$ or greater was achieved. Representative images were obtained using Koehler illumination with an Olympus Microfire digital camera (M/N S97809) attached to an Olympus BX60 microscope.

\section{Results}

Differentiation protocols I and II using retrovirally reprogammed cell lines

We compared retrovirally reprogammed cell lines A1145A and F1098A differentiation to DE by either Activin A treatment (Protocol I) or Myostatin (GDF8), GSK-3 $\beta$ inhibitor (CHIR99021) and B27 supplement (Protocol II). Both protocols resulted in similar expression of DE markers SOX 17 and CXCR4 (shown for F1098A cells, Figure 1A). The starting cell culture density was reported to be critical for successful HIO is [4]; however, SOX17 and CXCR4 expression was similar across a wide range of pre-differentiation \% confluent cells using both protocols and there was no correlation of initial confluency to the expression of either SOX17 $\left(r^{2}=0.06 ; p=0.27\right)$ or CXCR4 $\left(r^{2}=0.04 ; p=0.36\right)$.

Further incubation in KGF and RA produced robust expression of hindgut marker CDX2, as reported previously $[4,5]$ and this could be visualized in what appeared to be developing spheroids (arrows Figure 1B). Using either protocol, both A1145A (Figure 1C) and F1089A (Figure1D) produced easily excisable spheroids on top of the confluent layer of cells or hollow structures (Figure 1C, left panel), or floating spheroids (Figure 1D, left panel). Excisable spheroid structures that were transferred to 3D intestinal matrigel suspensions proliferated and formed structures that could be propagated over multiple "passages". The appearance of stage $3 \mathrm{HIO}$ derived by Protocol I and II from both cell lines was notably different. In the case of Protocol I HIO have dense core structures and spindly outgrowths (Figures 1C and 1D, top right panels), whereas Protocol II HIO exhibited dense core structures and many peripheral folded buds (Figures 1C and 1D, bottom right panels).

$\mathrm{HIO}$ derived by protocol I and II were also compared by immunohistochemical staining for the localization and intensity of the E-cadherin (epithelial cell adhesion complexes [25]), CDX2 (promotes hindgut intestinal cell fate [26], and villin (actin-binding protein associated with the intestinal brush border [27]. Antibody staining was optimized in banked human intestinal tissue (Figure 2A) and compared using the same staining protocol for $5 \mu \mathrm{m}$ sections from A1145A (Figure 2B)
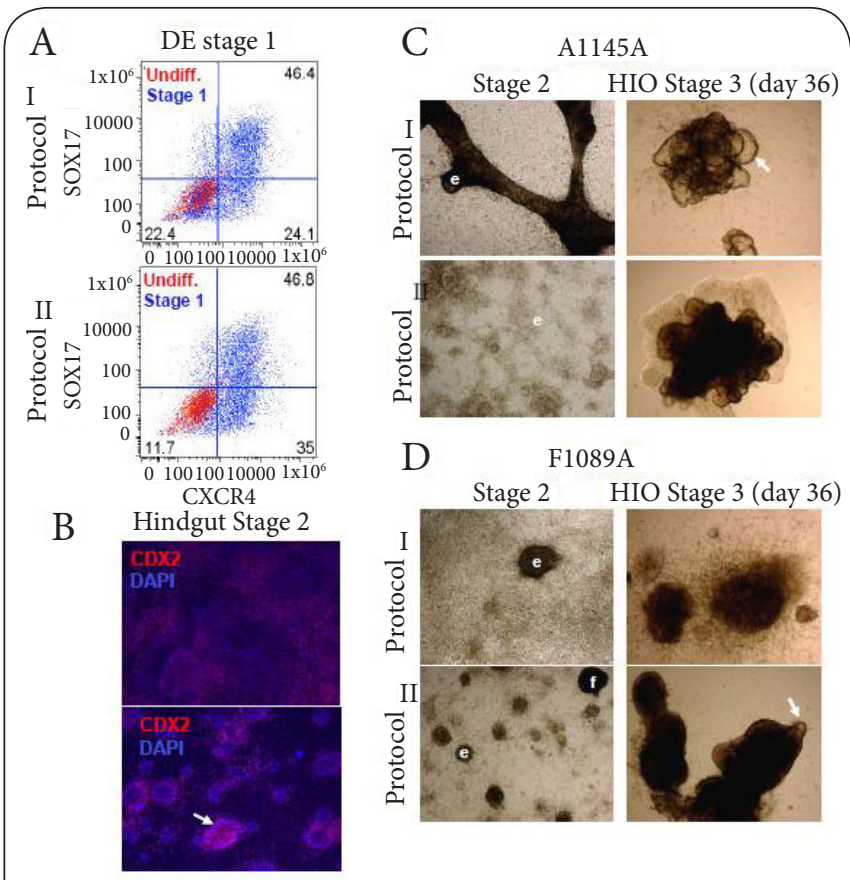

Figure 1. A: iPSCs express DE markers SOX17 and CXCR4 to a similar extent when differentiated using Protocol I (Activin A) or Protocol II (GDF8+GSK3i+B27), using F1089A cell line as an example. B: Hindgut CDX2 positive immunostaining is expressed in developing cellular clumps (spheroids) after protocol II incubation (KGF and RA) where nuclear (DAPI blue) and CDX2 (red) -immunoreactivity overlap (example shown by arrow) C: Representative images of morphology at different stages of A1145A. Protocol I (top) showed large elongated and dense structures at Stage 2, excised with some budding hollow structures that were (e) that were excised. Protocol II (bottom) showed spheroids at Stage 2 (e), In both protocols, HIO developed with a large number of budding hollow spheroids (e.g., arrow) during differentiation in 3D matrigel culture. 4X magnification. D: Representative images of morphology at different stages of F1089A. Protocol I at Stage 2 resulted in large compact spheroids that could be excised (e) and developed into dense HIO with ill-defined outgrowths during Stage 3 differentiation in 3D matrigel Protocol II (bottom) showed spheroids at Stage 2, which developed into HIO with a large number of budding hollow structures that were excisable (e) as well as and floating (f) spheroid structures. $4 \mathrm{X}$ magnification.

derived HIO. This confirmed differences in Protocol I and II using both cell lines derived $\mathrm{HIO}$ from excised spheroids. A1145A protocol I derived HIO showed weak E-cadherin immunoreactivity, however they did not express CDX2 or villin (Figures $2 \mathrm{~B}$ and $\mathbf{2 C}$ upper panel). In contrast, Protocol II excised spheroids from A1145A derived cell line (Figure 2A lower panel) developed into $\mathrm{HIO}$ with strong E-cadherin staining in cells, brush border villin localization and nuclear CDX2, apparently colocalized in the same cells by comparing adjacent sections.

The improvement in epithelial cell marker staining using protocol II was confirmed by F1089A-cell line derived HIO (Figure 3). 

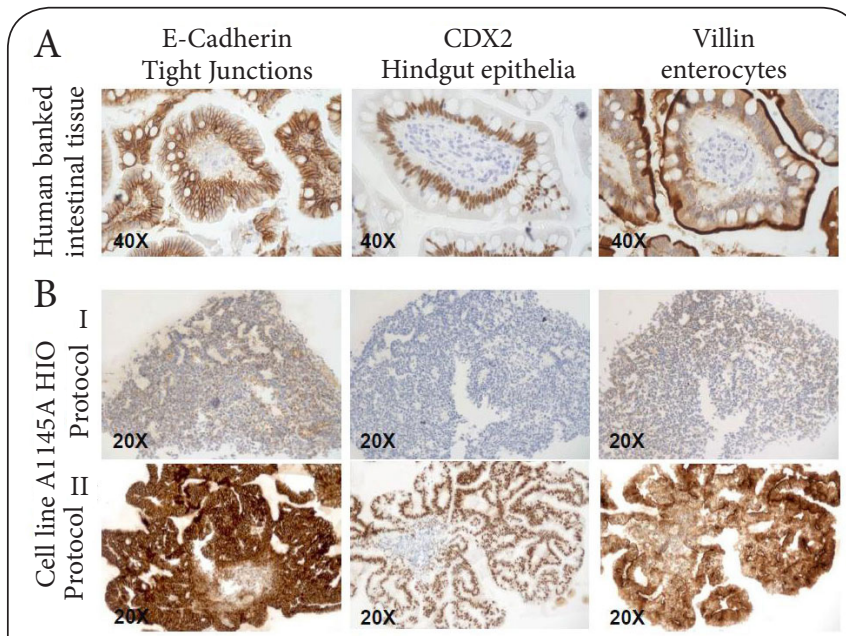

Figure 2. Immunostaining of intestinal epithelial marker expression in tissue and A1145A iPSC-derived HIO.

A: E-cadherin, CDX2 and villin staining in banked human intestinal tissue.

B: Protocol I HIO from excised stage 1 spheroids express low levels of iffuse staining of E-cadherin and villin, but not CDX2 after 36 days of Stage 3 differentiation.

$\mathrm{HIO}$-derived by protocol I showed some level of of E-cadherin and villin immunostaining but with weaker or patchier staining, and less convincing cell localization (Figure 3A upper panel) compared to the stronger expression of these markers and including CDX2 using protocol II (Figure 3B middle panel). In addition, We directly compared excised spheroids differentiated to $\mathrm{HIO}$ (as above) to the reported floating spheroids differentiated to HIO [4]. Protocol II-treated F1089A cell-derived stage 2 cells produced floating spheroids from the underlying adherent layer of cells (as shown in Figure 1D).

Immunohistochemistry of Protocol II-derived floating spheroids collected Stage 2 showed that these cells did not express intestinal markers (Figure 3B bottom panel). Thus, for iPSC lines A1145A and F1089A, the most promising Stage 3 $\mathrm{HIOs}$ were produced from Protocol II using excised spheroids at Stage 2. Overall successful spheroid formation seems critical for future successful differentiation. In addition to excised spheroids in matrigel being predictive appropriate HIO markers, we observed that the absence of CXCR4 at stage 1 resulted in absence of spheroids at Stage 2 (Figure 3B).

In addition to absorptive enterocytes, we visualized the enteroendocrine marker chromogranin A [28] in $\mathrm{HIO}$. Immunostaining was optimized in banked human intestinal tissue and A1145A-derived $\mathrm{HIO}$ showed a similar expression of chromogranin A in discrete puncta within $\mathrm{HIOs}$ (Figure 3C).

Multiple cell lines differentiate to HIO using protocol II Protocol II was used to compare different somatic cell types and reprogrammed iPSC lines (Table 1) to form HIO under similar differentiation conditions. Both retro-virally reprogrammed cell lines (A1145A and D2043A), and non-retro-virally

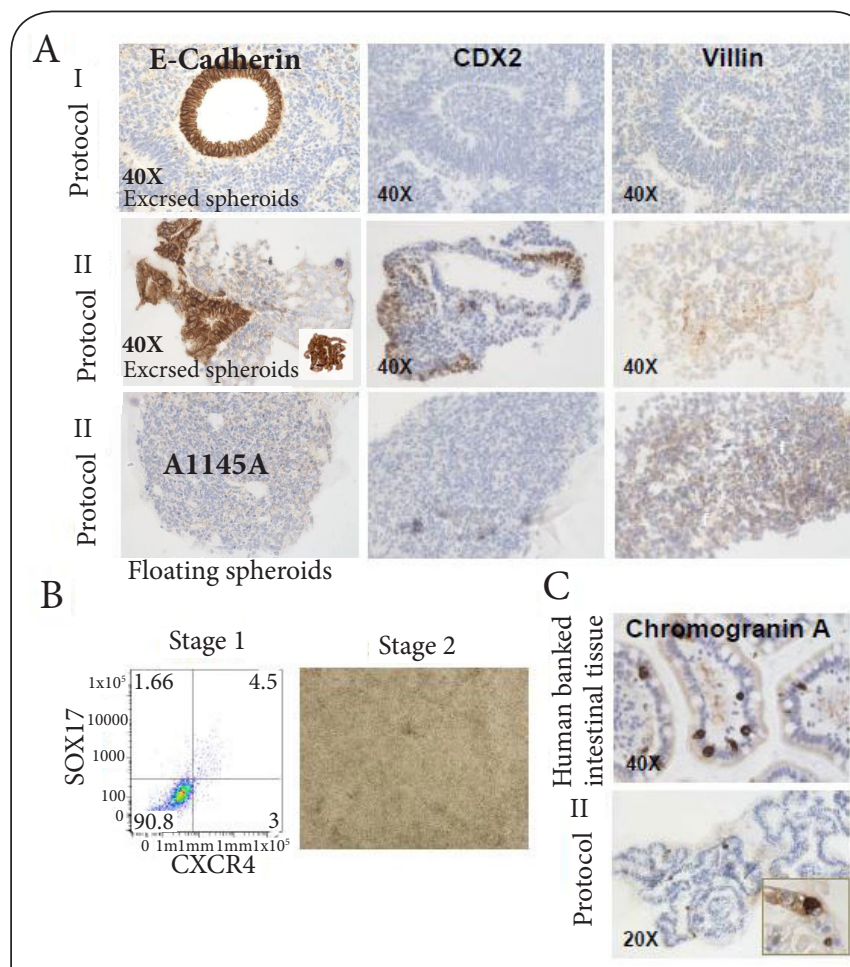

Figure 3. A: Immunostained HIO derived from F1089A excised spheroids by Protocol I and II excised spheroids (top and middle panel) and protocol II floating spheroids (bottom panel). Only Protocol II excised stage 2 spheroids develop into HIO that express moderate- intense immunostaining for E-cadherin, CDX2 and villin after 36 days of Stage 3 differentiation.

B: Stage 1 definitive endoderm marker expression is required for later stage morphology. Example of an iPSC line with poor definitive endoderm marker expression at Stage 1 that did not develop excisable spheroids by Stage 2.4X magnification. C: Enteroendoendocrine marker chromogranin A expression in human tissue (top) and HIO from A1145A differentiation (bottom).

reprogrammed cell lines (B2198A, C2128A and E2055A) were concurrently induced for intestinal organogenesis. Karyotype analysis was performed on all cell lines and F1089A cell line was discontinued for further study due to abnormal chromosome appearance in this cell line.

There was no apparent difference by flow cytometry analysis (Figure 4) between all pluripotent cell lines for expression of standard pluripotent markers $[29,30]$ indicating that all cell lines have similar differentiation potential. Specifically, all undifferentiated iPSC lines showed high expression (>70\%) of pluripotent markers, including surface markers SSEA3 and TRA-1-81, as well as transcription factors OCT4, SOX2, and Nanog. Cell lines did not express general differentiation marker SSEA1.

To compare DE formation at Stage 1, differentiating iPSClines were evaluated by flow cytometry for the expression of CXCR4 and SOX17. A1145A, B2198A, C2128A, D2043A, and 
Kauffman et al. Stem Cell Biology and Research 2015,

http://www.hoajonline.com/journals/pdf/2054-717X-2-1.pdf

doi: 10.7243/2054-717X-2-1

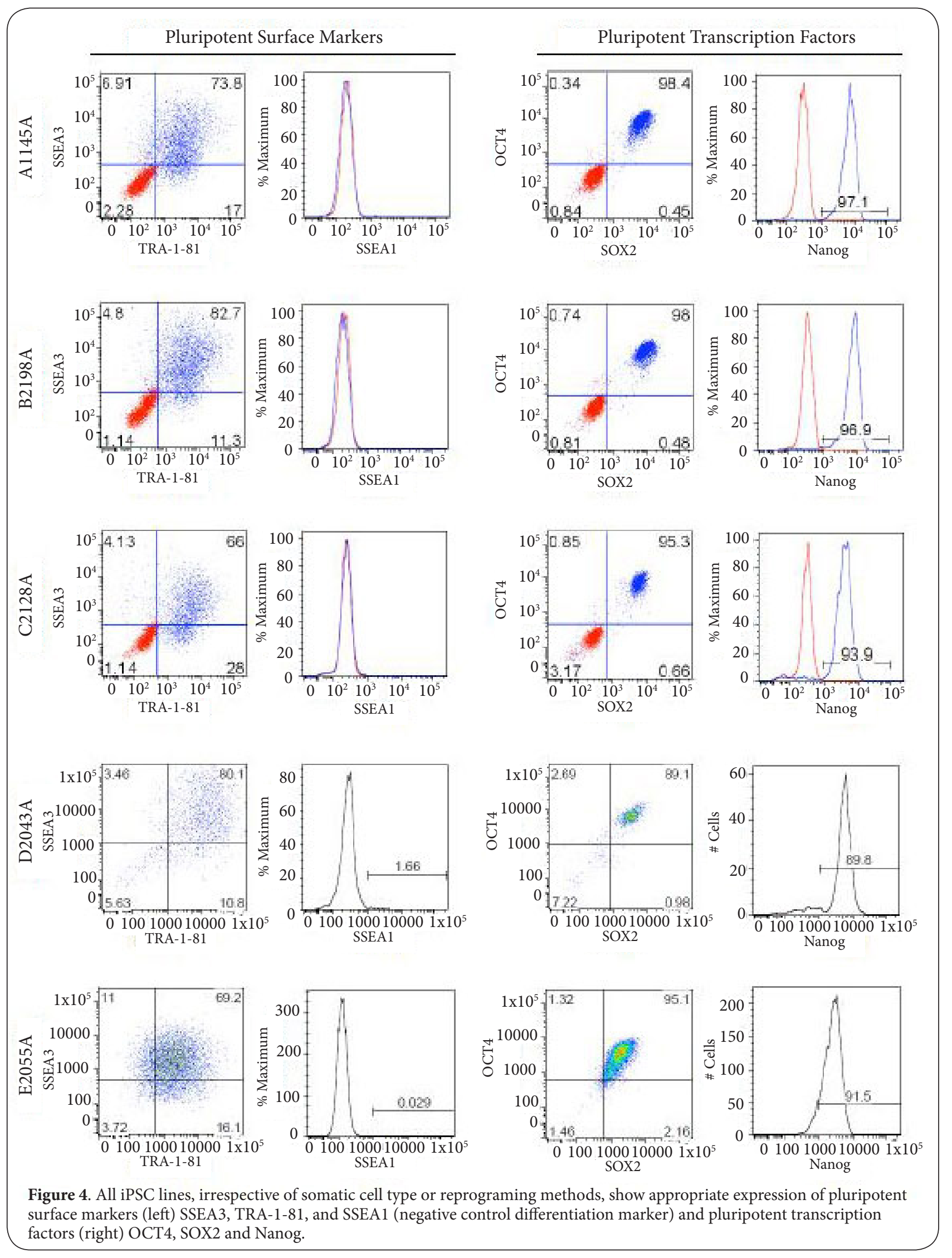


E2055A all exhibited double-positive expression of definitive endoderm cell surface receptor CXCR4 and transcription factor SOX 17 at Stage 1 to some extent, ranging from $42-73 \%$ of the iPSC-derived Stage1 population (Figure 5, right column). Undifferentiated C2128A had surprisingly high CXCR4 and SOX17 expression, but all the other cell lines had DE marker expression in Stage 1 that was generally much higher than that seen in undifferentiated cell populations (Figure 5, left column).

The morphological changes during differentiation of iPSC lines followed a similar general pattern (Figure 6). Differentiation to Stage 1 resulted in monolayer-like morphology for all iPSC lines (Figure 6, left column), with the appearance of developing dense structures in some instances. At Stage 2, cells within all iPSC-derived adherent cultures formed excisable spheroids at various sizes and frequency (Figure 6, middle column), which were collected and further differentiated in Stage 3 matrigel. After culture for 25 days dense structures formed that proliferated over multiple passages. Similar to the initial observations comparing Protocols I and II, HIO morphology differed by the amount of folded buds, reminiscent primary tissue intestinal crypts, and by the presence of long spindly outgrowths (Figure 6 right column). Semi-quantitative analysis of the formation of buds in Stage 3 indicated that there was a range of 3-15 buds per $\mathrm{HIO}$.

All IPSC-derived $\mathrm{HIO}$ were immunostained for E-cadherin, CDX2 and villin and qualitatively, all iPSC lines showed expression of E-cadherin, $\mathrm{CDX} 2$, and villin in at least a subset of cells (Figure 7). For example, expression E-cadherin did not appear to differ greatly in HIO similar intensity and cellular organization was noted, but regions of staining were less frequent in cell line E2055A. This cell line also had low expression of CDX2 and villin in the adjacent sections, both qualitatively and semi-quantitatively (Figure 7 left, bottom panel). Overall, the frequency and intensity of expression for CDX2 and villin was markedly greater for the two retrovirally reprogrammed cell lines, A1145A and D2043A (Figure 7 middle and right, bottom panel). These results suggest that, while this intestinal organogenesis protocol is capable of differentiating pluripotent stem cells from multiple sources into intestinal marker-expressing cells at Stage 3, some iPSC lines show more robust differentiation than others.

The amount of bud formation in Stage $3 \mathrm{HIO}$ was compared to the semi-quantitative immunohistochemical scoring for intestinal markers and was correlative in some cases. For example, E2055A, a cell line with low villin immunostaining at Stage 3, showed few formed buds, with a high incidence of spindly outgrowths. Supporting this, a correlation was noted between the average number of buds and villin expression in $\mathrm{HIO}$ across all cell lines $\left(r^{2}=0.79 ; p=0.04\right)$. On the other hand, no relationship between bud formation was noted for e-cadherin $\left(r^{2}=0.23 ; p=0.41\right)$ or $\operatorname{CDX} 2\left(r^{2}=0.35 ; p=0.29\right)$.

\section{Discussion and conclusion}

Two protocols for differentiating retrovirally reprogrammed
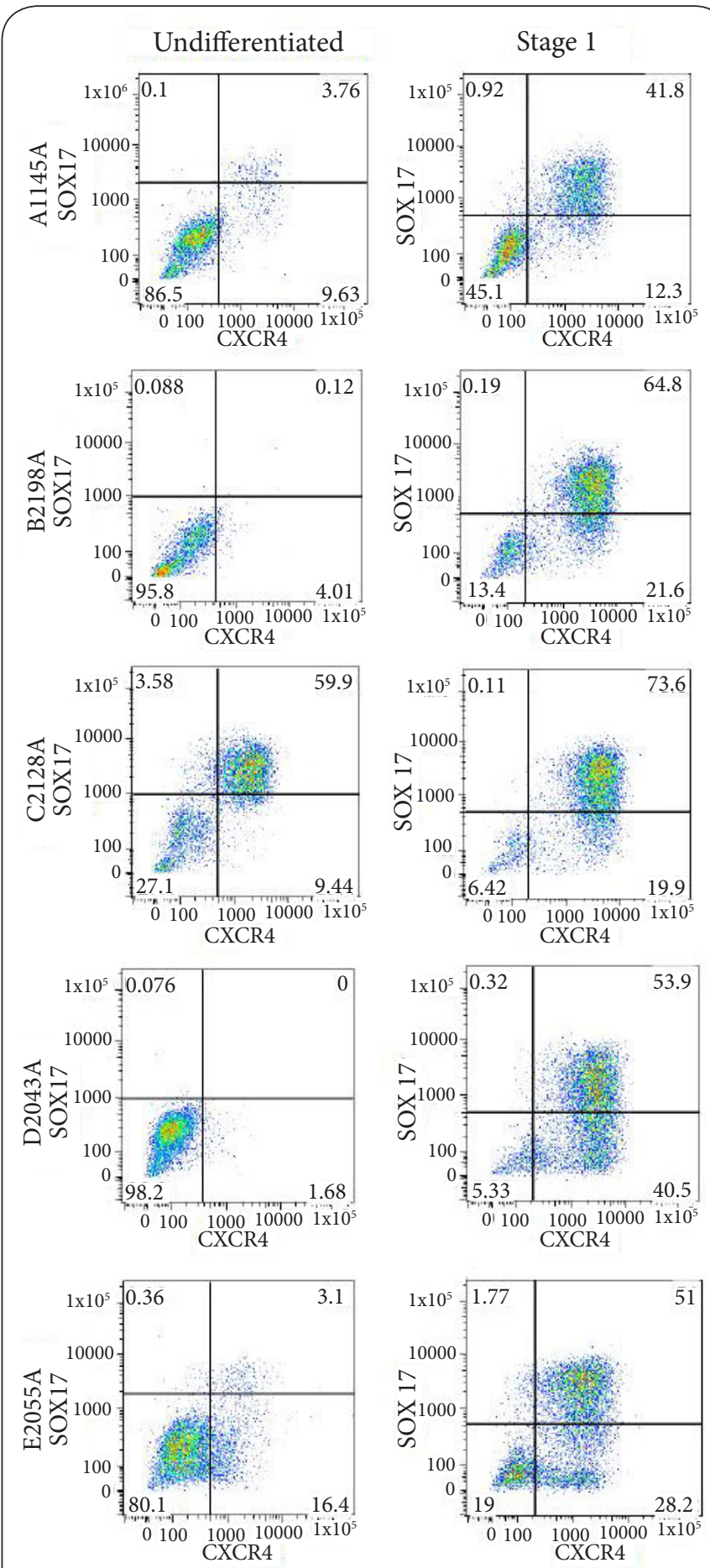

Figure 5. Expression of DE markers SOX17 and CXCR4 in iPSCs before differentiation and at Stage 1 assessed by flow cytometry. All iPSC lines showed similar increased expression of SOX17 and CXCR4 in Stage 1 except C2128A that had surprisingly high expression in Stage 1.

iPSCs to HIOs both resulted in equivalent differentiation to DE where cell expression of SOX17 and CXCR4 was indistinguishable. In protocol II GDF8, also in the TGF $\beta$ family, was 


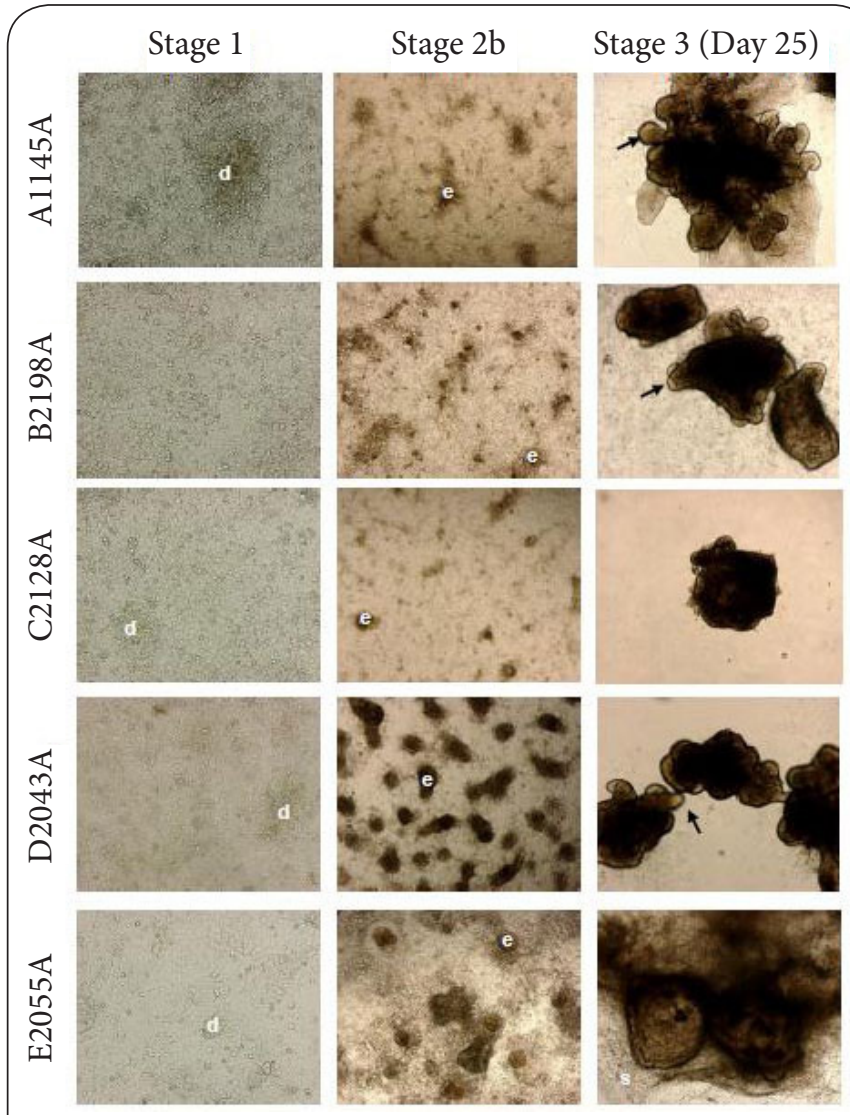

Figure 6. All iPSC stage-specific morphology. Left: At stage 1 , all lines showed monolayer morphology with sporadic dense structures ("d"). Middle: At stage 2, all lines developed excisable spheroids ("e"). Right: Excised spheroids all developed into Stage $3 \mathrm{HIO}$ with peripheral buds (arrows). Stage 1: 10X magnification; Stages 2 and 3: 4X magnification.

substituted for Activin A, and its robustness was also more cost-effective. Several morphological characteristics (excisable versus floating hindgut spheroids and extent of budding) were associated with successful hindgut differentiation into $\mathrm{HIO}$. These $\mathrm{HIO}$ had expression of intestinal epithelial markers E-Cadherin, CDX2, Villin, and Chromogranin A with some of the typical tissue architecture of adult intestine, although they are highly heterogeneous possibly because of a lack of embryonic axis formation [31].

Finally, both cell lines retrovirally reprogrammed and all three cell lines non-retrovirally reprogrammed from different somatic cells all produced $\mathrm{HIO}$. However, the retro-virally reprogrammed cells produced more robust $\mathrm{HIO}$ epithelial cell marker expression and this may be relevant for their use as translational 3D models of the gut physiology and pathophysiology. While retrovirally reprogrammed cell lines have been essential for the initial characterization of iPSCs relative to embryonic stem cell lines, they are incompatible with therapeutic applications, as genomic integration of reprogramming factors introduces a risk of insertional

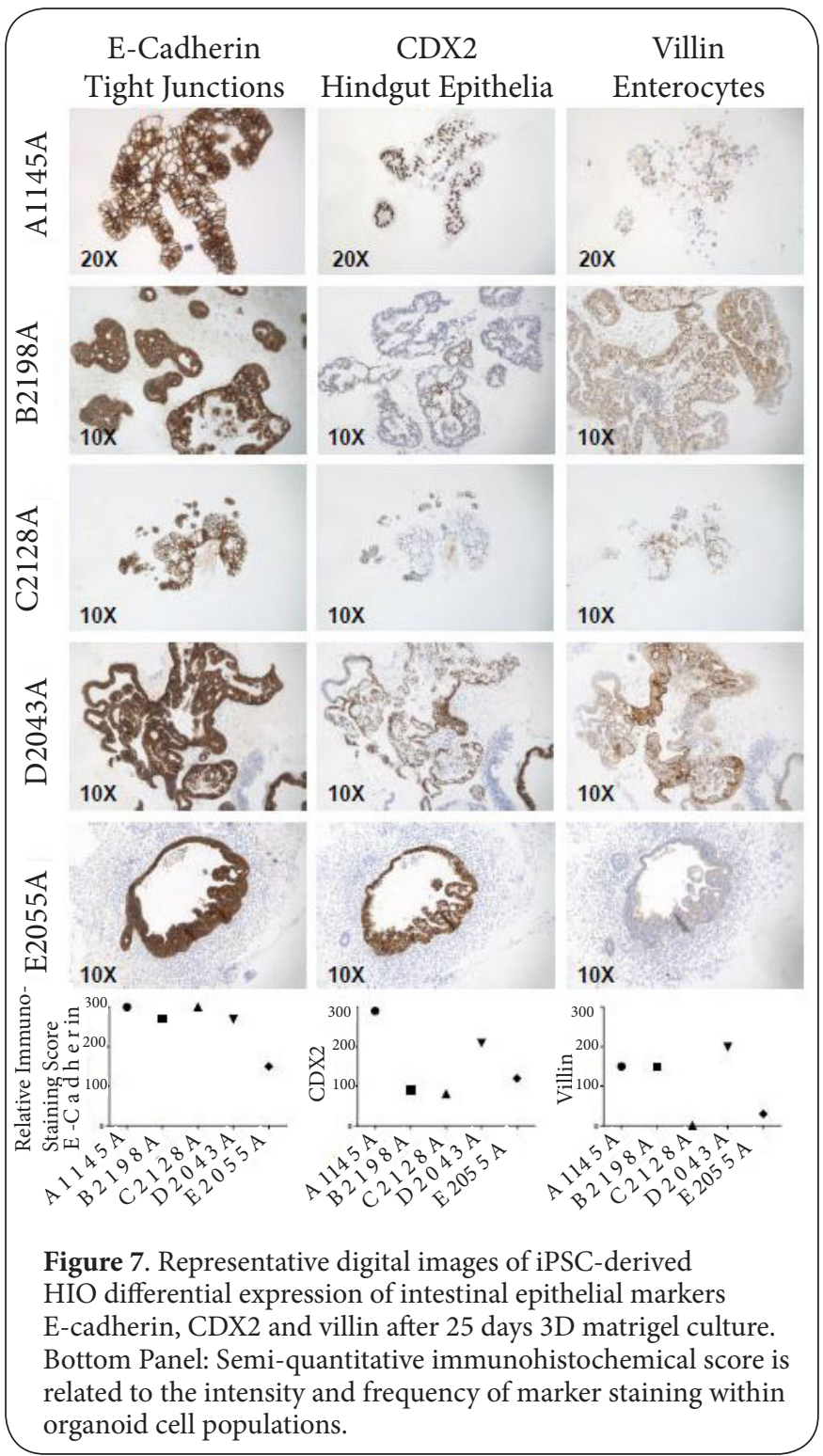

mutation that can potentially disrupt cellular stability and function [19]. High expression of pluripotent markers was evident in E2055A, which showed deviations from ideal ESlike morphology within maintenance cultures. Regenerative medicine approaches may be more appropriate by direct differentiation of crypt stem cell enteroids [32,33] rather than iPSC. Overall, these data can help improve cost-effectiveness, and ease of differentiation from different reprogrammed cell lines, enabling $\mathrm{HIO}$ to become more commonly available to research labs in this field.

Optimizing and understanding at least some early markers of successful HIO differentiation is important because of the time and effort it takes to make 3D HIO and their standardization. Furthermore the emergence of technology for in vivo models of human intestine generated from $\mathrm{HIO}$ engrafted into 
rodents require even more time and the use of animals [6].

As pointed out in a recent review, different methods used to generate intestinal organoids and maintain their growth in vitro, can confound comparisons from different studies [7]. We previously demonstrated that iPSC-derived intestinal cells express more physiologically relevant markers of epithelial lineages than another in vitro model of human intestinal epithelia such as immortalized cell line Caco-2 but limitations on the functionality such as barrier function remain [8]. Thus, the appropriate identification of robust $\mathrm{HIO}$ will be critical for reducing animal use for engrafted $\mathrm{HIO}$, which is required for the functionality and appropriate maturation of the epithelium and mesenchyme [6].

We did confirmed that differentiation protocols (Protocol I; $[4,5]$ and Protocol II [8] differentiated to DE and hindgut with excisable spheroids that can develop into 3D HIO. However, floating spheroids, in our hands, did not express CXCR4 nor develop into $\mathrm{HIO}$, as opposed to that reported $[4,5]$. Protocol I-differentiated A1145A or F1089A developed large elongated or spheroid structures attached to the adherent layer of differentiating cells and Protocol II-derived cells gave rise to mostly spheroid structures within the adherent layer of cells. In the case of iPSC line F1089A, floating spheroids did emerge by Stage 2, but immunohistochemical analysis revealed no expression of intestinal markers E-Cadherin, CDX2, or Villin in these floating spheroids. Particularly, the lack of CDX2 expression in the floating spheroids developed by Stage 2 of Protocol II intestinal organogenesis suggests that they were not precursors of intestinal organoids, as were those previously reported [5]. This was confirmed by immunohistochemistry at Stage 3, which showed that only excisable spheroids went on to produce $\mathrm{HIOs}$ with dense, budding morphology reminiscent of intestinal organoids derived from primary tissue intestinal crypts or intestinal stem cells [34], and intense expression of intestinal markers E-Cadherin, CDX2, and Villin.

Anther important observation was the pluripotent stem cell culture density upon initiation of differentiation. A higher starting cell density was previously reported to be critical for successful intestinal organogenesis [4]. However, there was no apparent difference in DE differentiation efficiency, when quantified in the present study by CXCR4 marker expression, within a range (30-100\%) of starting confluency of iPSC cell clumps.

Finally, we noted the chromogranin A, a marker for hormone-secreting enteroendocrine cells was expressed much earlier in our protocol at Day 28; whereas it was previously reported to be present in $\mathrm{HIO}$ after $>100$ days in culture [5]. Enteroendocrine function such as incretin release will be valuable for studying microbiome product-intestinal interactions [35]. Overall, these studies confirm that generating $\mathrm{HIO}$ is a viable option for producing intestinal epithelial-like cells from human iPSCs. It provides a perspective on key parameters to avoid or look out for, with an advantage of cost-effectiveness of this protocol relative to previously published methods.
Competing interests

The authors declare that they have no competing interests.

Authors' contributions

\begin{tabular}{|l|c|c|c|c|c|}
\hline Authors' contributions & ALK & JEK & AVG & MAR & PJH \\
\hline Research concept and design & $\checkmark$ & -- & - & $\checkmark$ & $\checkmark$ \\
\hline Collection and/or assembly of data & $\checkmark$ & $\checkmark$ & $\checkmark$ & -- & -- \\
\hline Data analysis and interpretation & $\checkmark$ & $\checkmark$ & $\checkmark$ & -- & -- \\
\hline Writing the article & $\checkmark$ & -- & -- & -- & $\checkmark$ \\
\hline Critical revision of the article & $\checkmark$ & -- & $\checkmark$ & -- & $\checkmark$ \\
\hline Final approval of article & -- & -- & -- & -- & $\checkmark$ \\
\hline Statistical analysis & -- & -- & -- & -- & $\checkmark$ \\
\hline
\end{tabular}

\section{Acknowledgement}

We thank Charito Buensuceso, Mark Zimmerman and Lubing Zhou for help in obtaining in-house iPSC lines, as well as Nathan Majewski and Kimberly Reese for help with iPSC maintenance culture. We also acknowledge the immunohistochemistry performed by Steve Bernstein and Marko Srdanov, Qualtek Molecular Labs, Goleta, CA. All investigators are employees of Johnson \& Johnson and have no other conflict of interest to disclose.

\section{Publication history}

Editor: Kiminobu Sugaya, University of Central Florida, USA.

EIC: Prasad S. Koka, Haffkine Institute for Training, Research \& Testing, India.

Received: 24-Dec-2014 Final Revised: 24-Jan-2015

Accepted: 16-Feb-2015 Published: 23-Feb-2015

\section{References}

1. Takahashi K, Tanabe K, Ohnuki M, Narita M, Ichisaka T, Tomoda K and Yamanaka $S$. Induction of pluripotent stem cells from adult human fibroblasts by defined factors. Cell. 2007; 131:861-72. | Article I PubMed

2. Chen YJ, Finkbeiner SR, Weinblatt D, Emmett MJ, Tameire F, Yousefi M, Yang C, Maehr R, Zhou Q, Shemer R, Dor Y, Li C, Spence JR and Stanger $B Z$. De novo formation of insulin-producing "neo-beta cell islets" from intestinal crypts. Cell Rep. 2014; 6:1046-58. | Article | PubMed Abstract I PubMed Full Text

3. Finkbeiner $S R$ and Spence JR. A gutsy task: generating intestinal tissue from human pluripotent stem cells. Dig Dis Sci. 2013; 58:1176-84. Article I PubMed Abstract | PubMed Full Text

4. McCracken KW, Howell JC, Wells JM and Spence JR. Generating human intestinal tissue from pluripotent stem cells in vitro. Nat Protoc. 2011; 6:1920-8. | Article | PubMed Abstract | PubMed Full Text

5. Spence JR, Mayhew CN, Rankin SA, Kuhar MF, Vallance JE, Tolle K, Hoskins EE, Kalinichenko VV, Wells SI, Zorn AM, Shroyer NF and Wells JM. Directed differentiation of human pluripotent stem cells into intestinal tissue in vitro. Nature. 2011; 470:105-9. | Article | PubMed Abstract | PubMed Full Text

6. Watson CL, Mahe MM, Munera J, Howell JC, Sundaram N, Poling HM, Schweitzer JI, Vallance JE, Mayhew CN, Sun Y, Grabowski G, Finkbeiner SR, Spence JR, Shroyer NF, Wells JM and Helmrath MA. An in vivo model of human small intestine using pluripotent stem cells. Nat Med. 2014; 20:1310-4. | Article | PubMed

7. Wells JM and Spence JR. How to make an intestine. Development. 2014; 141:752-60. | Article | PubMed

8. Kauffman AL, Gyurdieva AV, Mabus JR, Ferguson C, Yan Z and Hornby PJ. Alternative functional in vitro models of human intestinal epithelia. Front Pharmacol. 2013; 4:79. | Article | PubMed Abstract | PubMed Full Text

9. D'Amour KA, Agulnick AD, Eliazer S, Kelly OG, Kroon E and Baetge EE. Efficient differentiation of human embryonic stem cells to definitive 
Kauffman et al. Stem Cell Biology and Research 2015,

http://www.hoajonline.com/journals/pdf/2054-717X-2-1.pdf

doi: 10.7243/2054-717X-2-1

endoderm. Nat Biotechnol. 2005; 23:1534-41. | Article | PubMed

10. Norrman K, Strombeck A, Semb H and Stahlberg A. Distinct gene expression signatures in human embryonic stem cells differentiated towards definitive endoderm at single-cell level. Methods. 2013; 59:5970. | Article | PubMed

11. Dessimoz J, Opoka R, Kordich JJ, Grapin-Botton A and Wells JM. FGF signaling is necessary for establishing gut tube domains along the anterior-posterior axis in vivo. Mech Dev. 2006; 123:42-55. | Article | PubMed

12. McLin VA, Rankin SA and Zorn AM. Repression of Wnt/beta-catenin signaling in the anterior endoderm is essential for liver and pancreas development. Development. 2007; 134:2207-17. | Article | PubMed

13. Plateroti M, Freund JN, Leberquier $C$ and Kedinger M. Mesenchymemediated effects of retinoic acid during rat intestinal development. $J$ Cell Sci. 1997; 110 ( Pt 10):1227-38. | Article | PubMed

14. McCormack SA, Viar MJ, Tague $L$ and Johnson LR. Altered distribution of the nuclear receptor RAR beta accompanies proliferation and differentiation changes caused by retinoic acid in Caco-2 cells. In Vitro Cell Dev Biol Anim. 1996; 32:53-61. I PubMed

15. Visco V, Bava FA, d'Alessandro F, Cavallini M, Ziparo V and Torrisi MR. Human colon fibroblasts induce differentiation and proliferation of intestinal epithelial cells through the direct paracrine action of keratinocyte growth factor. J Cell Physiol. 2009; 220:204-13. | Article | PubMed

16. Visco V, Belleudi F, Marchese C, Leone L, Aimati L, Cardinali G, Kovacs $D$, Frati $L$ and Torrisi MR. Differential response to keratinocyte growth factor receptor and epidermal growth factor receptor ligands of proliferating and differentiating intestinal epithelial cells. J Cell Physiol. 2004; 200:31-44. | Article | PubMed

17. Houle $M$, Prinos P, Iulianella $A$, Bouchard $N$ and Lohnes D. Retinoic acid regulation of $\mathrm{Cdx1}$ : an indirect mechanism for retinoids and vertebral specification. Mol Cell Biol. 2000; 20:6579-86. | Article I PubMed Abstract I PubMed Full Text

18. Silberg DG, Swain GP, Suh ER and Traber PG. Cdx1 and cdx2 expression during intestinal development. Gastroenterology. 2000; 119:961-71. | Article I PubMed

19. Bayart $E$ and Cohen-Haguenauer O. Technological overview of iPS induction from human adult somatic cells. Curr Gene Ther. 2013; 13:7392. | Article | PubMed Abstract | PubMed Full Text

20. Kauffman AL, Gyurdieva AV, Mabus JR and Hornby PJ. Human Intestinal Epithelium Derived From Induced Pluripotent Stem Cells. Gastroenterology. 2013; 144:S1-33.

21. Alder J, Kramer BC, Hoskin C and Thakker-Varia S. Brain-derived neurotrophic factor produced by human umbilical tissue-derived cells is required for its effect on hippocampal dendritic differentiation. Dev Neurobiol. 2012; 72:755-65. | Article | PubMed

22. Yakubov E, Rechavi G, Rozenblatt $S$ and Givol D. Reprogramming of human fibroblasts to pluripotent stem cells using mRNA of four transcription factors. Biochem Biophys Res Commun. 2010; 394:189-93. | Article | PubMed

23. Jia F, Wilson KD, Sun N, Gupta DM, Huang M, Li Z, Panetta NJ, Chen ZY, Robbins RC, Kay MA, Longaker MT and Wu JC. A nonviral minicircle vector for deriving human iPS cells. Nat Methods. 2010; 7:197-9. | Article | PubMed Abstract | PubMed Full Text

24. Ladner RD, Lynch FJ, Groshen S, Xiong YP, Sherrod A, Caradonna SJ, Stoehlmacher J and Lenz HJ. dUTP nucleotidohydrolase isoform expression in normal and neoplastic tissues: association with survival and response to 5-fluorouracil in colorectal cancer. Cancer Res. 2000; 60:3493-503. | Article | PubMed

25. Zbar AP, Simopoulos C and Karayiannakis AJ. Cadherins: an integral role in inflammatory bowel disease and mucosal restitution. J Gastroenterol. 2004; 39:413-21. | Article I PubMed

26. Gao N, White $P$ and Kaestner KH. Establishment of intestinal identity and epithelial-mesenchymal signaling by Cdx2. Dev Cell. 2009; 16:58899. | Article | PubMed Abstract | PubMed Full Text

27. Friederich $E$, Vancompernolle $K$, Louvard D and Vandekerckhove J. Villin function in the organization of the actin cytoskeleton. Correlation of in vivo effects to its biochemical activities in vitro. J Biol Chem. 1999; 274:26751-60. | Article | PubMed

28. O'Connor DT, Burton D and Deftos LJ. Chromogranin A: immunohistology reveals its universal occurrence in normal polypeptide hormone producing endocrine glands. Life Sci. 1983; 33:1657-63. | Article PubMed

29. Alvarez CV, Garcia-Lavandeira M, Garcia-Rendueles ME, Diaz-Rodriguez E, Garcia-Rendueles AR, Perez-Romero S, Vila TV, Rodrigues JS, Lear PV and Bravo SB. Defining stem cell types: understanding the therapeutic potential of ESCs, ASCs, and iPS cells. J Mol Endocrinol. 2012; 49:R89111. | Article | PubMed

30. Ohnishi H, Oda Y, Aoki T, Tadokoro M, Katsube Y, Ohgushi H, Hattori K and Yuba S. A comparative study of induced pluripotent stem cells generated from frozen, stocked bone marrow- and adipose tissuederived mesenchymal stem cells. J Tissue Eng Regen Med. 2012; 6:26171. | Article | PubMed

31. Lancaster MA and Knoblich JA. Organogenesis in a dish: modeling development and disease using organoid technologies. Science. 2014; 345:1247125. | Article | PubMed

32. Petersen N, Reimann F, Bartfeld S, Farin HF, Ringnalda FC, Vries RG, van den Brink S, Clevers $H$, Gribble FM and de Koning EJ. Generation of $L$ cells in mouse and human small intestine organoids. Diabetes. 2014; 63:41020. | Article | PubMed Abstract | PubMed Full Text

33. Sato $T$ and Clevers H. Growing self-organizing mini-guts from a single intestinal stem cell: mechanism and applications. Science. 2013; 340:1190-4. | Article | PubMed

34. Sato $T$, Vries RG, Snippert $H J$, van de Wetering $M$, Barker $N$, Stange $D E$, van Es JH, Abo A, Kujala P, Peters PJ and Clevers H. Single Lgr5 stem cells build crypt-villus structures in vitro without a mesenchymal niche. Nature. 2009; 459:262-5. | Article | PubMed

35. Lukovac S, Belzer C, Pellis L, Keijser BJ, de Vos WM, Montijn RC and Roeselers G. Differential modulation by Akkermansia muciniphila and Faecalibacterium prausnitzii of host peripheral lipid metabolism and histone acetylation in mouse gut organoids. MBio. 2014; 5. | Article | PubMed Abstract | PubMed Full Text

Citation:

Kauffman AL, Ekert JE, Gyurdieva AV, Rycyzyn MA and Hornby PJ. Directed differentiation protocols for successful human intestinal organoids derived from multiple induced pluripotent stem cell lines. Stem Cell Biol Res. 2015; 2:1.

http://dx.doi.org/10.7243/2054-717X-2-1 